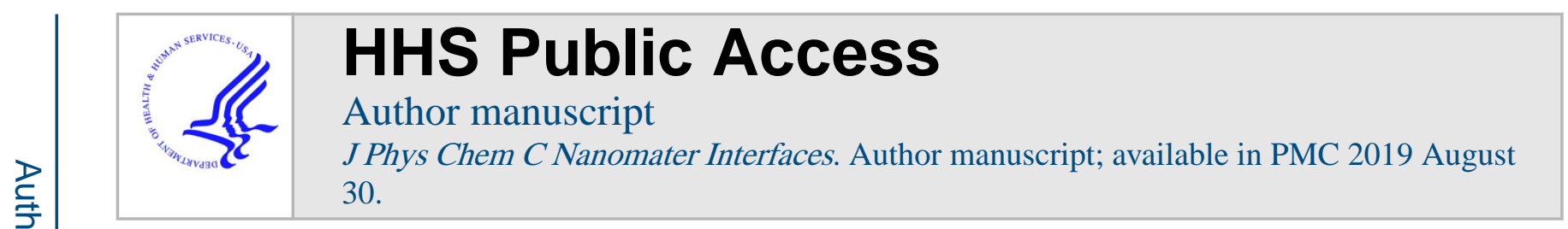

Published in final edited form as:

J Phys Chem C Nanomater Interfaces. 2018 August 30; 122(34): 19699-19704. doi:10.1021/acs.jpcc. 8 b06117.

\title{
Near-Infrared Photothermal Release of siRNA from the Surface of Colloidal Gold-Silver-Gold Core-Shell-Shell Nanoparticles Studied with Second-Harmonic Generation
}

\author{
Raju R. Kumal ${ }^{\dagger}$, Mohammad Abu-Laban ${ }^{\ddagger}$, Prakash Hamal ${ }^{\dagger}$, Blake Kruger $^{\dagger}$, Holden T. \\ Smith $^{\dagger}$, Daniel J. Hayes ${ }^{\ddagger}$, and Louis H. Haber ${ }^{*}, \dagger$ \\ tDepartment of Chemistry, Louisiana State University, Baton Rouge, Louisiana 70803, United \\ States \\ ¥Department of Biomedical Engineering, The Pennsylvania State University, University Park, \\ Pennsylvania 16802, United States
}

\begin{abstract}
Photothermal release of oligonucleotides from the surface of plasmonic nanoparticles represents a promising platform for spatiotemporal controlled drug delivery. Here we demonstrate the use of novel gold-silver-gold core-shell-shell (CSS) nanoparticles to study the photothermal cleaving and release of micro-RNA (miRNA) mimics or small interfering RNA (siRNA) under nearinfrared (NIR) irradiation. The furan-maleimide-based Diels-Alder adduct cleaves thermally above $60{ }^{\circ} \mathrm{C}$ and is used to bind siRNA to the colloidal nanoparticle surface in water. We investigate the photothermal cleaving kinetics of siRNA under different NIR laser powers using surface-sensitive time-dependent second-harmonic generation (SHG) spectroscopy. The photothermal release of siRNA from the surface of CSS nanoparticles is significantly higher than that from the surface of gold nanoparticles (GNPs) under similar experimental conditions. These results demonstrate that plasmonic CSS nanoparticles with photothermal cleaving linkers have important potential applications for nanoparticle-based NIR-mediated drug-delivery systems.
\end{abstract}

\section{Graphical Abstract}

\footnotetext{
*Corresponding Author lhaber@ @ lsu.edu. Tel: (225) 578-7965.

Notes

The authors declare no competing financial interest.

ASSOCIATED CONTENT

Supporting Information

The Supporting Information is available free of charge on the ACS Publications website at DOI: 10.1021/acs.jpcc.8b06117.

Details of the synthesis of the different plasmonic nanoparticles used and additional characterization measurements from transmission electron microscopy, electrophoresis, dynamic light scattering, and SHG timeresolved studies (PDF)
} 


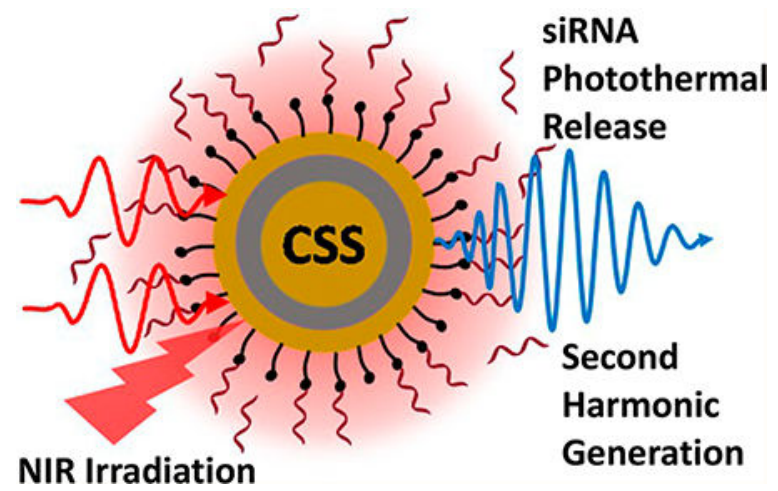

\section{INTRODUCTION}

Thermally cleaving linkers enable nanoparticle-based systems in which molecular and biological cargo can be delivered to target sites under spatial and temporal control. ${ }^{1,2}$ Whereas other methods of release such as $\mathrm{pH}$-induced release ${ }^{3}$ and liposome encapsulation ${ }^{4}$ have been studied, thermal cleavage can be induced by a wider range of external stimuli sources that are capable of releasing thermal energy, either directly by bulk heating or indirectly by photothermal or magnetic hysteresis processes. ${ }^{5,6}$ Recent investigations described the use of retro-Diels-Alder-based chemistry to induce thermal cleavage using a furan and maleimide cycloadduct group to tether a fluorophore to gold nanoshell surfaces. ${ }^{7}$ Cleavage at $60{ }^{\circ} \mathrm{C}$ was demonstrated with water bath experiments, and additional studies reported success with similar linker mimics. ${ }^{8}$ Here we report the synthesis of gold-silvergold core-shell- shell (CSS) nanoparticles linked to a siRNA mimic of miRNA148b using a furan-maleimide-based Diels-Alder adduct for photothermal release studies under nearinfrared irradiation monitored in real time using second-harmonic generation spectroscopy.

Plasmonic nanoparticles composed of gold and silver have been widely studied due to their potential applications in nanomedicine, catalysis, photovoltaics, and optoelectronics. ${ }^{9-13}$ These nanoparticles have unique properties that include localized surface plasmon resonances, large surface area to volume ratios, low toxicities, excellent biocompatibilities, and chemical control of associated surface functionalities. ${ }^{14-18}$ Localized surface plasmon resonances are coherent oscillations of free electrons excited by electromagnetic radiation that depend on the size, shape, composition, and surrounding medium of the nanoparticles. 12,19-21 Additionally, the surface chemistry and synthesis methods of gold and silver nanoparticles are compatible for the preparation of hybrid and bimetallic core-shell nanoparticles. ${ }^{22}$ Bimetallic core-shell nanoparticles have drawn special interest because of their altered optical and electrical properties as compared with the corresponding monometallic nanoparticles. By controlling the size and ratio of the core and shell dimensions, the resulting plasmonic spectra of the core-shell nanoparticles can be tuned and optimized for desired plasmonic applications. ${ }^{23,24}$

In our previous work, we investigated the photocleaving kinetics of miRNA mimics from the surface of gold and silver nanoparticles with nitrobenzyl linkers using second-harmonic generation spectroscopy under ultraviolet irradiation. ${ }^{25,26}$ We also studied the retro-Diels- 
Alder thermal release of siRNA from the surface of silver nanoparticles under varying temperatures. ${ }^{27}$ To extend photoactivated drug delivery to near-infrared wavelengths that are better suited for the optical water window of biological samples, novel gold-silver-gold CSS nanoparticles are used in this current study. The plasmon peak of these CSS nanoparticles is in the NIR region, and the peak can be adjusted by changing the relative core and shell sizes. ${ }^{22}$ The siRNA is attached to the surface of the CSS nanoparticles using a thermally cleavable bicyclic linker made through the Diels-Alder reaction of furfuryl mercaptan with 6maleimidohexanoic acid, as previously described. ${ }^{27}$ Upon photothermal excitation in the NIR region, a retro-Diels- Alder reaction is initiated when the local temperature around the particle reaches $\sim 60{ }^{\circ} \mathrm{C}$, releasing the siRNA payload. The kinetics of the photothermal release is monitored in real time using second-harmonic generation. The fluorophore Alexa350 is attached to the $5^{\prime}$ end of the siRNA for additional quantification measurements. The Scheme 1 shows the siRNA attachment and release from a CSS nanoparticle surface using Diels-Alder chemistry.

Second-harmonic generation is a powerful, noninvasive, surface-sensitive technique that is useful for the investigation of colloidal nanoparticles. ${ }^{28-35}$ In SHG spectroscopy, two incident photons of frequency $\omega$ add coherently to generate a photon of frequency $2 \omega$. This nonlinear optical process is dipole forbidden in bulk media with inversion symmetry. However, SHG can be produced from the surface of nanoparticles where the inversion symmetry is broken. The second-harmonic field $\mathrm{E}_{\mathrm{SHG}}$ is proportional to the square root of the SHG intensity, $I_{\mathrm{SHG}}$, given by

$$
\sqrt{I_{\mathrm{SHG}}}=E_{\mathrm{SHG}}=\chi^{(2)} E_{\omega}^{2}+\chi^{(3)} E_{\omega}^{2} \Phi_{0}
$$

where, $\chi^{(2)}$ and $\chi^{(3)}$ are second- and third-order nonlinear susceptibilities, respectively, $E_{\omega}$ is the electric field of the incident laser with frequency $\omega$, and $\Phi_{0}$ is the electrostatic surface potential. $32,36-42$

\section{EXPERIMENTAL SECTION}

\section{A. Synthesis and Characterization.}

The preparation of the siRNA-functionalized gold-silver-gold CSS nanoparticles and the siRNA-functionalized GNPs is described in the Supporting Information. The colloidal nanoparticle samples are centrifuged and resuspended in ultrapure water before and after siRNA functionalization for the SHG measurements. The nanoparticles are characterized using extinction spectroscopy, dynamic light scattering (DLS), zeta potential measurements, and transmission electron microscopy (TEM). Figure 1a shows a representative TEM image of CSS nanoparticles with an average size of $120 \pm 15 \mathrm{~nm}$. Figure $1 \mathrm{~b}$ shows a TEM image at higher magnification of a CSS nanoparticle functionalized with siRNA (siRNA-CSS). The average hydrodynamic diameter of the siRNA-functionalized CSS nanoparticles is measured to be $148 \pm 50 \mathrm{~nm}$ with a polydispersity index of 0.2 using DLS. The larger size of the functionalized particle as measured by DLS is due to the additional hydrodynamic radius generated by the siRNA extending out into solution. The electrophoretic mobilities of the 
CSS nanoparticles and siRNA-functionalized CSS nanoparticles are measured to be $-2.3 \times$ $10^{-8}$ and $-3.1 \times 10^{-8} \mathrm{~m}^{2} /(\mathrm{V} \mathrm{s})$, respectively. The corresponding zeta potentials of the CSS nanoparticles and siRNA-functionalized CSS nanoparticles are $-44.7 \pm 10.2$ and -59.3 $\pm 12.3 \mathrm{mV}$, respectively, where the increased zeta potential magnitude is caused by the added negative surface charge density from the siRNA. Additional details including TEM images of GNP seeds, gold-silver core-shell (CS) nanoparticles, and GNPs are shown in the Supporting Information. The plasmon peak of the GNP seeds is blue-shifted upon the addition of the silver shell and then red-shifted upon the addition of the outer gold shell in forming the final structure, as shown in Figure 2. The plasmon peaks of the GNP seeds, the gold-silver CS nanoparticles, and the gold-silver-gold CSS nanoparticles are centered at 524,420 , and $745 \mathrm{~nm}$, respectively.

\section{B. Experimental Setup.}

The experimental setup for monitoring the photothermal release of siRNA from the surface of colloidal CSS nanoparticles using SHG has been previously described. ${ }^{25,26} \mathrm{~A}$ titanium:sapphire oscillator laser centered at $800 \mathrm{~nm}$ with a 70 fs pulse duration, $80 \mathrm{MHz}$ repetition rate, and $2.6 \mathrm{~W}$ average output power is used. The SHG signal is collected in the forward direction using a highsensitivity charged-coupled device (CCD) spectroscopy detector coupled to the monochromator spectrograph. A portion of the oscillator laser beam passes through a beam splitter to seed the amplifier laser. The amplifier produces a laser beam centered at $800 \mathrm{~nm}$ with a $75 \mathrm{fs}$ pulse duration, a $10 \mathrm{kHz}$ repetition rate, and a $7 \mathrm{~W}$ average output power. The amplifier laser is used to irradiate the CSS nanoparticle sample to induce the photothermal cleaving of the siRNA, while the remaining portion of the oscillator laser is used to monitor the photothermal cleaving kinetics in real time using SHG spectroscopy. A computer-controlled beam block is used to block the amplifier irradiation beam while taking SHG signal with the oscillator laser. For the SHG measurements, the oscillator laser power is attenuated to a constant value of $300 \mathrm{~mW}$ to minimize photothermal release from the CSS surface while probing the kinetics. A $1 \mathrm{~cm} \times 1 \mathrm{~cm}$ quartz cuvette is used to contain $2 \mathrm{~mL}$ of the nanoparticle sample. The amplifier laser power is varied, and the photothermal cleaving kinetics are monitored as a function of irradiation time and power.

\section{RESULTS AND DISCUSSION}

Representative SHG spectra of siRNA-functionalized CSS nanoparticles at different NIR irradiation times of 0, 1.5, and $5 \mathrm{~min}$ are shown in Figure 3. The SHG signal is centered at $400 \mathrm{~nm}$ with a full width at half-maximum of $4.5 \mathrm{~nm}$. The slight rise in intensity at longer wavelengths is attributed to two-photon fluorescence from the nanoparticles.

Oligonucleotides attached to the surface of the nanoparticles increase the SHG signal due to the high surface charge density through the $\chi^{(3)}$ effect. ${ }^{25,26}$ The SHG signal from the siRNA-functionalized CSS sample decreases with time under NIR irradiation due to photothermal release of siRNA from the nanoparticle surface.

Figure 4a shows the power-dependent analysis of the photothermal release of siRNA from the surface of CSS nanoparticles. The sample is irradiated with different laser powers of 0 , $20,50,100$, and $200 \mathrm{~mW}$ centered at $800 \mathrm{~nm}$ using the amplifier laser. The time trace using 
no NIR irradiation from the amplifier laser $(0 \mathrm{~mW})$ shows only a slight decrease in SHG intensity of $\sim 1 \%$ over time, which is attributed to a small amount of photothermal cleaving by the oscillator probe laser. The kinetics of the photothermal release of siRNA under NIR irradiation is analyzed by fitting the SHG time traces using a pseudo-first-order exponential function given by $E_{\mathrm{SHG}}=A+B e^{-k t}$, where $A$ and $B$ are proportionality constants. ${ }^{25,26}$ The obtained rate constants $\mathrm{k}$ are $(0.03 \pm 0.15) \times 10^{-2},(0.78 \pm 0.06) \times 10^{-2},(1.12 \pm 0.06) \times 10^{-2}$, $(1.77 \pm 0.04) \times 10^{-2}$, and $(1.85 \pm 0.06) \times 10^{-2} \mathrm{~s}^{-1}$ for $0,20,50,100$, and $200 \mathrm{~mW}$ irradiation laser powers, respectively. The obtained rate constants are plotted as a function of laser power, as shown in Figure 4b. Interestingly, the ultrafast amplifier NIR irradiation pulses cause much higher photothermal cleaving rates than the ultrafast oscillator laser at comparable average powers. This may be caused by hot electrons leading to higher photothermal efficiencies on ultrafast time scales ${ }^{43,44}$ which are predicted to scale with pulse power rather than average laser power. This suggests that the pulse duration and repetition rate are both important factors for optimizing photothermal and drug-delivery applications, although more research is needed to study this process in more detail. The photothermal release of siRNA from the surface of $105 \mathrm{~nm}$ GNPs is also studied under different NIR irradiation powers, with the results shown in the Supporting Information. A much larger decrease in SHG intensity for the CSS nanoparticles indicates a much higher release of siRNA from the surface of CSS nanoparticles in comparison with GNPs due to the enhanced photothermal effect, in agreement with fluorescence quantification measurements, as described later.

Figure 5 shows results from control experiments where the variation of the SHG intensity as a function of the NIR irradiation time is displayed for the CSS and the siRNA-functionalized CSS nanoparticles. The SHG signal from the CSS nanoparticle sample remains constant over time using the $800 \mathrm{~nm}$ probe laser with $300 \mathrm{~mW}$ average power, indicating a stable SHG signal for the CSS nanoparticle sample. However, the SHG intensity signal from the siRNA-functionalized CSS nanoparticle sample decreases and asymptotically reaches a minimum value when using both the probe laser and the pump laser with $200 \mathrm{~mW}$ power centered at $800 \mathrm{~nm}$. The minimum SHG signal from the siRNA-CSS sample under NIR irradiation is approximately equal to the corresponding signal of the CSS nanoparticle, indicating complete photothermal release of oligonucleotides from the surface of nanoparticles. Here both the CSS and siRNA-functionalized CSS samples have a concentration of $\sim 8.3 \times 10^{7}$ nanoparticles $/ \mathrm{mL}$.

The thermal release of siRNA from the surface of colloidal CSS nanoparticles is also studied using SHG spectroscopy under varying solution temperatures. The thermal treatment consists of placing $2 \mathrm{~mL}$ of the siRNA-functionalized CSS sample in a quartz cuvette wrapped by electrical heating tape. The sample is allowed to run for 14 min under slow stirring for each measurement using temperatures of $25,40,60$, and $80^{\circ} \mathrm{C}$ while measuring the SHG signal as a function of time. Thermal release of siRNA from the surface of CSS nanoparticle is shown in Figure 6. There is no change in the SHG signal at 25 and $40{ }^{\circ} \mathrm{C}$, indicating that the oligonucleotides do not release at room temperature or normal body temperature. The rate increase of photothermal release is apparent at temperatures of 60 and $80{ }^{\circ} \mathrm{C}$ with faster cleavage rates at the higher temperature of $80^{\circ} \mathrm{C}$. Similarly, the thermal release of siRNA from the surface of GNP is also studied at different temperatures. The 
extent of thermal release of siRNA from the surface of GNP is comparable to that from the surface of CSS nanoparticles, as shown in the Supporting Information.

The amount of siRNA released from the surface of CSS nanoparticles is quantified using fluorescence measurements with the results displayed in Figure 7. The procedure for siRNA fluorescence quantification has been previously described. ${ }^{25,26}$ The nanoparticle samples are centrifuged, and the amount of remaining siRNA in the supernatant is quantified from a fluorescence calibration curve. The amount of siRNA released from the surface of CSS and GNPs is comparable when performing chemical reduction using dithiothreitol (DTT), which completely cleaves the siRNA from the nanoparticle surface. The coverage of siRNA to the CSS nanoparticle surface is determined to be $440 \pm 20$ oligo/ nanoparticle. Under NIR irradiation, the amount of siRNA released from the surface of siRNA-functionalized CSS nanoparticles is significantly higher than the amount released from the GNPs. The CSS nanoparticles show complete cleavage of siRNA using NIR irradiation for $9 \mathrm{~min}$ at powers of 20,50,100, and $200 \mathrm{~mW}$, respectively, to within experimental uncertainty. Much lower siRNA release is observed for the GNP sample under NIR irradiation, with increasing release under increasing NIR powers. These results further demonstrate the dramatically improved photothermal efficiencies and corresponding photothermal cleaving efficiencies of the siRNA-functionalized CSS nanoparticles compared with the siRNA-functionalized gold nanoparticles.

\section{CONCLUSIONS}

The NIR photothermal cleaving kinetics of siRNA functionalized to the surface of goldsilver-gold core-shell-shell nanoparticles are studied using time-dependent secondharmonic generation spectroscopy. The core and shell sizes are optimized for a maximum plasmonic peak in the NIR region, as shown by extinction spectroscopy and TEM measurements. The siRNA is attached to the CSS surface by a thermal-cleavable linker prepared by Diels-Alder chemistry. Additionally, fluorescence quantification of the amount of siRNA release shows that the photothermal cleaving efficiency is significantly higher for the CSS nanoparticles compared with gold nanoparticles prepared under similar conditions. These results demonstrate that gold-silver-gold core-shell-shell nanoparticles with photothermal cleaving linkers can be successfully utilized for targeted drug-delivery applications using near-infrared irradiation in the optical window of biological tissues.

\section{Supplementary Material}

Refer to Web version on PubMed Central for supplementary material.

\section{ACKNOWLEDGMENTS}

\footnotetext{
We thank Louisiana State University, the National Science Foundation (CBET-1254281 and OIA-1541079), and the National Institute Dental and Craniofacial Research of the National Institutes of Health (RDE024790A) for generous financial support. We also acknowledge Dr. Rafael Cueto for help with in DLS and zeta potential measurements and Ying Xiao from Material Characterization Center (LSUMCC) at LSU for help with TEM images.
} 


\section{REFERENCES}

(1). Cobley CM; Au L; Chen J; Xia Y Targeting Gold Nanocages to Cancer Cells for Photothermal Destruction and Drug Delivery. Expert Opin. Drug Delivery 2010, 7, 577-587.

(2). Mura S; Nicolas J; Couvreur P Stimuli-Responsive Nanocarriers for Drug Delivery. Nat. Mater. 2013, 12, 991-1003. [PubMed: 24150417]

(3). Gupta P; Vermani K; Garg S Hydrogels: From Controlled Release to pH-Responsive Drug Delivery. Drug Discovery Today 2002, 7, 569-579. [PubMed: 12047857]

(4). Balazs DA; Godbey W Liposomes for Use in Gene Delivery. J. Drug Delivery 2011, 2011, 1-12.

(5). Chen G-J; Wang L-F Design of Magnetic Nanoparticles-Assisted Drug Delivery System. Curr. Pharm. Des. 2011, 17, 2331-2351. [PubMed: 21736546]

(6). Thibaudau F Ultrafast Photothermal Release of DNA From Gold Nanoparticles. J. Phys. Chem. Lett 2012, 3, 902-907. [PubMed: 26286418]

(7). Bakhtiari ABS; Hsiao D; Jin G; Gates BD; Branda NR An Efficient Method Based on the Photothermal Effect for the Release of Molecules From Metal Nanoparticle Surfaces. Angew. Chem., Int. Ed. 2009, 48, 4166-4169.

(8). Yamashita S; Fukushima H; Niidome Y; Mori T; Katayama Y; Niidome T Controlled-Release System Mediated by a Retro Diels-Alder Reaction Induced by the Photothermal Effect of Gold Nanorods. Langmuir 2011, 27, 14621-14626. [PubMed: 21988322]

(9). Austin LA; Mackey MA; Dreaden EC; El-Sayed MA The Optical, Photothermal, and Facile Surface Chemical Properties of Gold and Silver Nanoparticles in Biodiagnostics, Therapy, and Drug Delivery. Arch. Toxicol 2014, 88, 1391-1417. [PubMed: 24894431]

(10). Haes AJ; Hall WP; Chang L; Klein WL; Van Duyne RP A Localized Surface Plasmon Resonance Biosensor: First Steps Toward an Assay for Alzheimer's Disease. Nano Lett. 2004, 4, 1029_ 1034.

(11). Hu M; Chen J; Li Z-Y; Au L; Hartland GV; Li X; Marquez M; Xia Y Gold Nanostructures: Engineering Their Plasmonic Properties for Biomedical Applications. Chem. Soc. Rev 2006, 35, 1084-1094. [PubMed: 17057837]

(12). Hutter E; Fendler JH Exploitation of Localized Surface Plasmon Resonance. Adv. Mater. 2004, $16,1685-1706$.

(13). Jackson J; Halas N Silver Nanoshells: Variations in Morphologies and Optical Properties. J. Phys. Chem. B 2001, 105, 2743-2746.

(14). Nikoobakht B; Wang J; El-Sayed MA Surface-Enhanced Raman Scattering of Molecules Adsorbed on Gold Nanorods: Off-Surface Plasmon Resonance Condition. Chem. Phys. Lett 2002, 366, 17-23.

(15). Olson J; Dominguez-Medina S; Hoggard A; Wang L-Y; Chang W-S; Link S Optical Characterization of Single Plasmonic Nanoparticles. Chem. Soc. Rev 2015, 44, 40-57. [PubMed: 24979351]

(16). Pattnaik P Surface Plasmon Resonance. Appl. Biochem. Biotechnol. 2005, 126, 079-092.

(17). Willets KA; Van Duyne RP Localized Surface Plasmon Resonance Spectroscopy and Sensing. Annu. Rev. Phys. Chem 2007, 58, 267-297. [PubMed: 17067281]

(18). Yeh Y-C; Creran B; Rotello VM Gold Nanoparticles: Preparation, Properties, and Applications in Bionanotechnology. Nanoscale 2012, 4, 1871-1880. [PubMed: 22076024]

(19). Halas NJ; Lal S; Chang W-S; Link S; Nordlander P Plasmons in Strongly Coupled Metallic Nanostructures. Chem. Rev 2011, 111, 3913-3961. [PubMed: 21542636]

(20). Klar T; Perner M; Grosse S; Von Plessen G; Spirk1 W; Feldmann J Surface-Plasmon Resonances in Single Metallic Nanoparticles. Phys. Rev. Lett 1998, 80, 4249-4252.

(21). Lal S; Link S; Halas NJ Nano-Optics from Sensing to Waveguiding. Nat. Photonics 2007, 1 , 641-648.

(22). Karam TE; Smith HT; Haber LH Enhanced Photothermal Effects and Excited-State Dynamics of Plasmonic Size-Controlled Gold-Silver-Gold Core-Shell-Shell Nanoparticles. J. Phys. Chem. C 2015, 119, 18573-18580. 
(23). Ghosh Chaudhuri R; Paria S Core/Shell Nanoparticles: Classes, Properties, Synthesis Mechanisms, Characterization, and Applications. Chem. Rev 2012, 112, 2373-2433. [PubMed: 22204603]

(24). Zhong C-J; Maye MM Core-Shell Assembled Nanoparticles as Catalysts. Adv. Mater 2001, 13, 1507-1511.

(25). Kumal RR; Abu-Laban M; Landry CR; Kruger B; Zhang Z; Hayes DJ; Haber LH PlasmonEnhanced Photocleaving Dynamics in Colloidal MicroRNA-Functionalized Silver Nanoparticles Monitored with Second Harmonic Generation. Langmuir 2016, 32, 10394-10401. [PubMed: 27605308]

(26). Kumal RR; Landry CR; Abu-Laban M; Hayes DJ; Haber LH Monitoring the Photocleaving Dynamics of Colloidal MicroRNA-Functionalized Gold Nanoparticles Using Second Harmonic Generation. Langmuir 2015, 31, 9983-9990. [PubMed: 26313536]

(27). Abu-Laban M; Kumal RR; Casey J; Becca J; LaMaster D; Pacheco CN; Sykes DG; Jensen L; Haber LH; Hayes DJ Comparison of Thermally Actuated Retro-Diels-Alder Release Groups for Nanoparticle Based Nucleic Acid Delivery. J. Colloid Interface Sci 2018, 526, 312-321. [PubMed: 29751265]

(28). Yan ECY; Liu Y; Eisenthal KB New Method for Determination of Surface Potential of Microscopic Particles by Second Harmonic Generation. J. Phys. Chem. B 1998, 102, 6331-6336.

29). Hayes PL; Malin JN; Jordan DS; Geiger FM Get Charged up: Nonlinear Optical Voltammetry for Quantifying the Thermodynamics and Electrostatics of Metal Cations at Aqueous/ Oxide Interfaces. Chem. Phys. Lett. 2010, 499, 183-192.

(30). Gonella G; Dai H-L Determination of Adsorption Geometry on Spherical Particles From Nonlinear Mie Theory Analysis of Surface Second Harmonic Generation. Phys. Rev. B: Condens. Matter Mater. Phys 2011, 84, 1214021-1214025.

(31. ) Gonella G; Dai H-L Second Harmonic Light Scattering from the Surface of Colloidal Objects: Theory and Applications. Langmuir 2014, 30, 2588-2599. [PubMed: 24171670]

(32). Kumal RR; Karam TE; Haber LH Determination of the Surface Charge Density of Colloidal Gold Nanoparticles Using Second Harmonic Generation. J. Phys. Chem. C 2015, 119, 1620016207.

(33). Kumal RR; Nguyenhuu H; Winter JE; McCarley RL; Haber LH Impacts of Salt, Buffer, and Lipid Nature on Molecular Adsorption and Transport in Liposomes As Observed by Second Harmonic Generation. J. Phys. Chem. C 2017, 121, 15851-15860.

(34). Doughty B; Yin P; Ma Y-Z Adsorption, Ordering, and Local Environments of SurfactantEncapsulated Polyoxometalate Ions Probed at the Air-Water Interface. Langmuir 2016, 32 , 8116-8122. [PubMed: 27452922]

(35). Tran RJ; Sly KL; Conboy JC Applications of Surface Second Harmonic Generation in Biological Sensing. Annu. Rev. Anal. Chem 2017, 10, 387-414.

(36). Eisenthal KB Second Harmonic Spectroscopy of Aqueous Nano-and Microparticle Interfaces. Chem. Rev 2006, 106, 1462-1477. [PubMed: 16608187]

(37. ) Dadap JI; Shan J; Heinz TF Theory of Optical Second-Harmonic Generation From a Sphere of Centrosymmetric Material: Small-Particle Limit. J. Opt. Soc. Am. B 2004, 21, 1328-1347.

(38). Geiger FM Second Harmonic Generation, Sum Frequency Generation, and $\chi$ (3): Dissecting Environmental Interfaces with a Nonlinear Optical Swiss Army Knife. Annu. Rev. Phys. Chem 2009, 60, 61-83. [PubMed: 18851705]

(39). tgebaucks C; Gonella G; Roke S. Optical Label-Free and Model-Free Probe of the Surface Potential of Nanoscale and Microscopic Objects in Aqueous Solution. Phys. Rev. B: Condens. Matter Mater. Phys 2016, 94, 195410.

(40. ) Rao Y; Kwok SJ; Lombardi J; Turro NJ; Eisenthal KB Label-Free Probe of HIV-1 TAT Peptide Binding to Mimetic Membranes. Proc. Natl. Acad. Sci. U. S. A 2014, 111, 12684-12688. [PubMed: 25136100]

(41. ) Subir M; Liu J; Eisenthal KB Protonation at the Aqueous Interface of Polymer Nanoparticles with Second Harmonic Generation. J. Phys. Chem. C 2008, 112, 15809-15812.

(42). Zhao X; Ong S; Wang H; Eisenthal KB New Method for Determination of Surface pKa Using Second Harmonic Generation. Chem. Phys. Lett 1993, 214, 203-207. 
(43). Goodman AM; Hogan NJ; Gottheim S; Li C; Clare SE; Halas NJ Understanding Resonant LightTriggered DNA Release From Plasmonic Nanoparticles. ACS Nano 2017, 11, 171-179. [PubMed: 28114757]

(44). Hatef A; Fortin-Deschenes S; Boulais E; Lesage F; Meunier M Photothermal Response of Hollow Gold Nanoshell to Laser Irradiation: Continuous Wave, Short and Ultrashort Pulse. Int. J. Heat Mass Transfer 2015, 89, 866-871. 


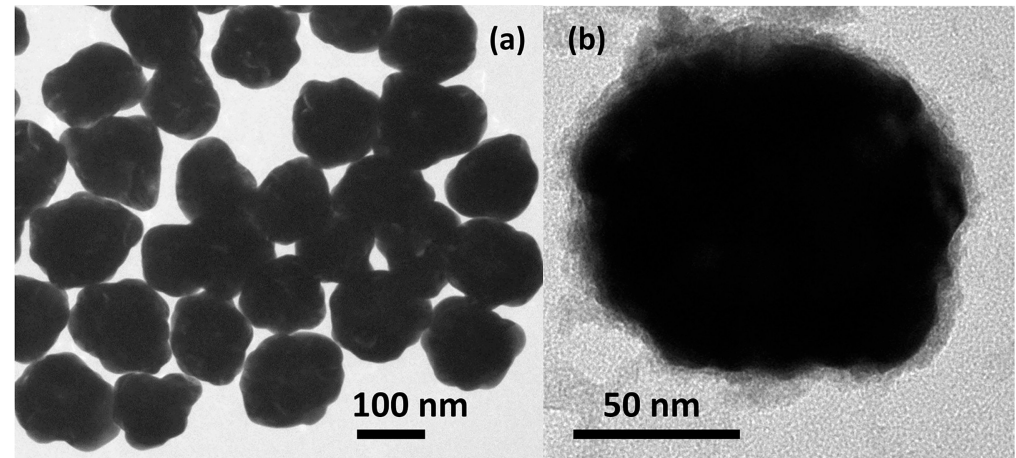

Figure 1.

Representative TEM images of (a) gold-silver-gold core- shell-shell nanoparticles and (b) siRNA-functionalized gold-silver-gold core-shell-shell nanoparticles. 


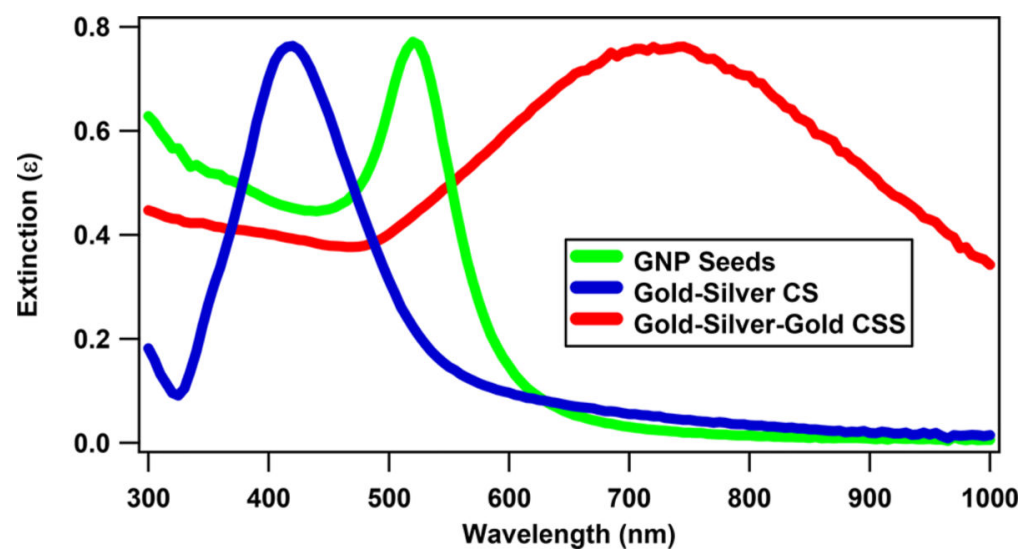

Figure 2.

Extinction spectra of gold nanoparticle seeds (green line), gold-silver core-shell nanoparticles (blue line), and gold-silver- gold core-shell-shell nanoparticles (red line). 


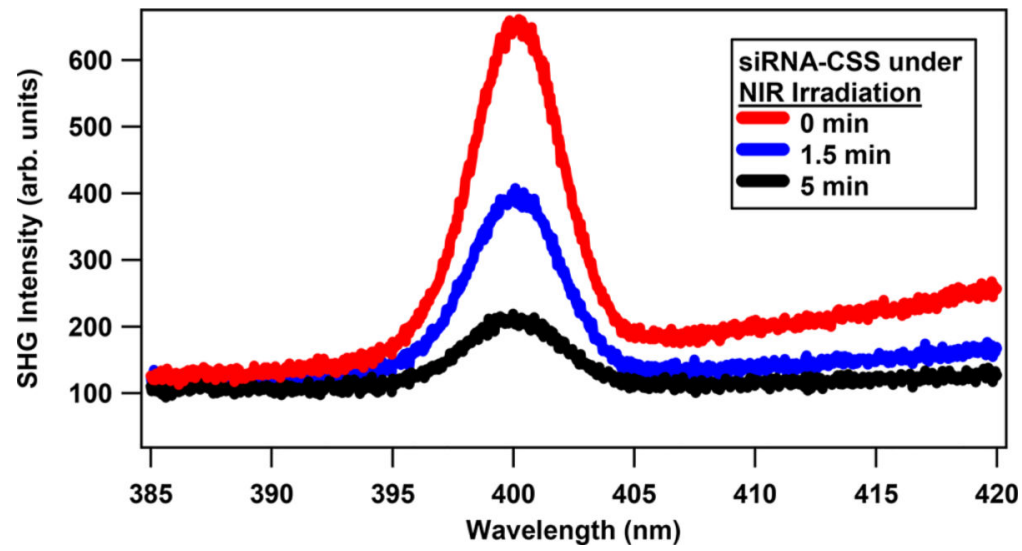

Figure 3.

Representative SHG spectra of siRNA-functionalized CSS nanoparticles at different NIR irradiation times. 

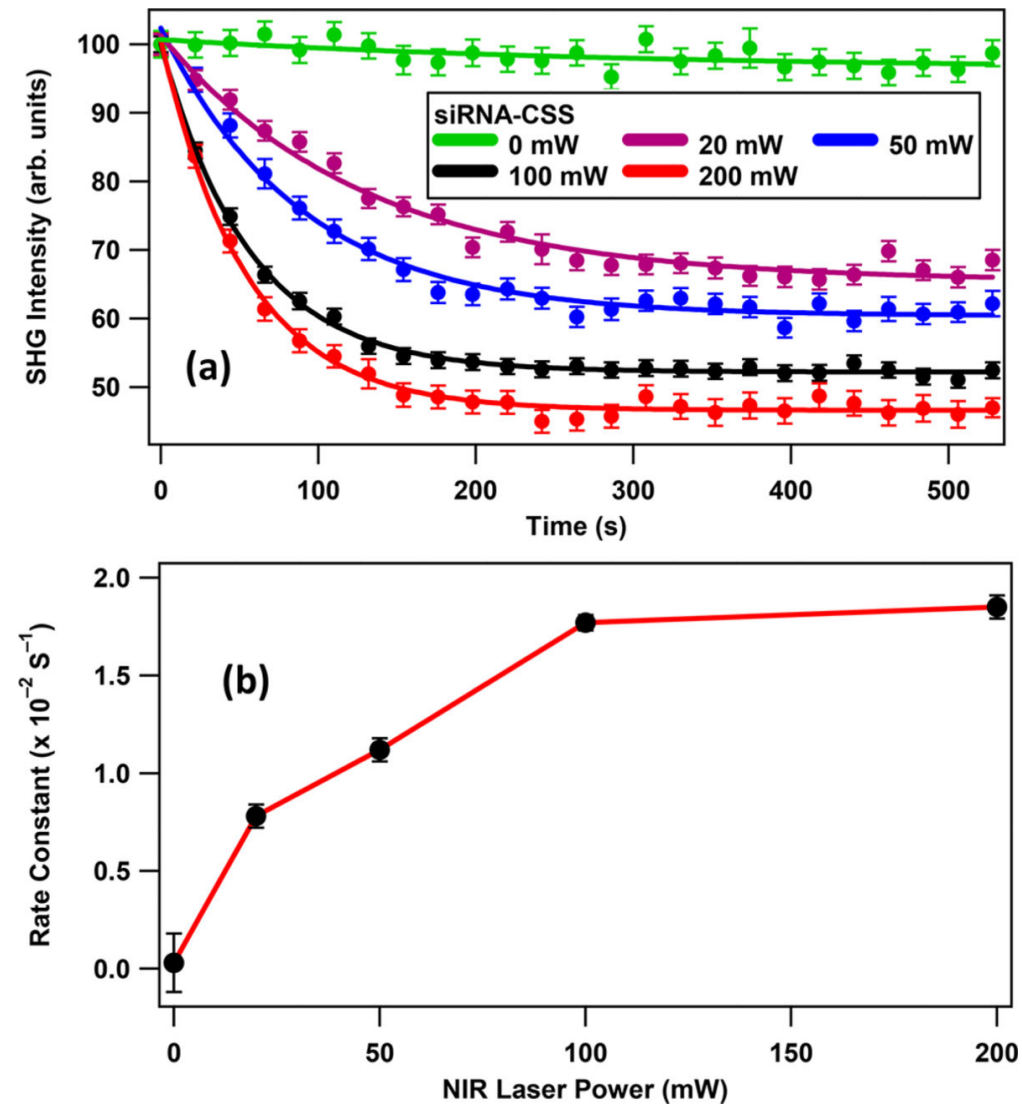

Figure 4.

(a) SHG intensity of the siRNA-functionalized CSS nanoparticles as a function of time under varying NIR irradiation powers. (b) Obtained photothermal cleaving rate constants as a function of laser power. 


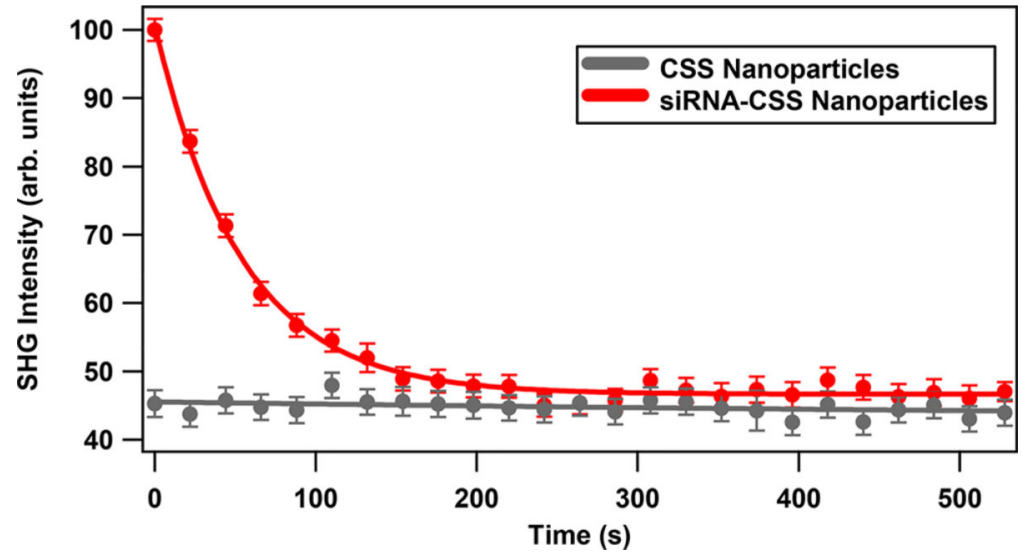

Figure 5.

Control experiments showing the time-dependent SHG signal for CSS nanoparticles using the probe laser only (gray data) and for the siRNA-functionalized CSS nanoparticles using both the probe laser and the $200 \mathrm{~mW}$ NIR irradiation laser (red data). 


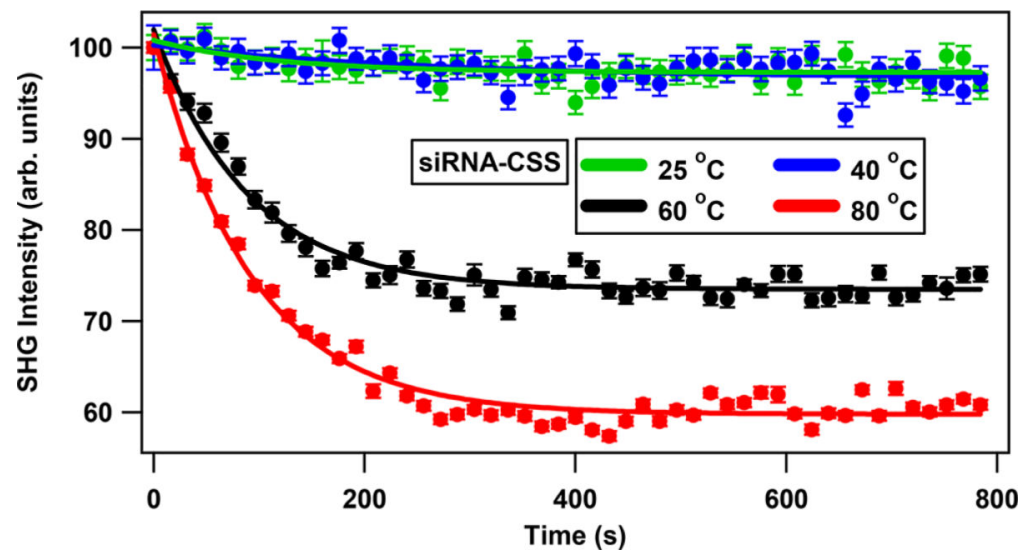

Figure 6.

SHG intensity as a function of time from the siRNA-functionalized CSS nanoparticles showing the thermal release of siRNA from the nanoparticle surface under different temperatures. 


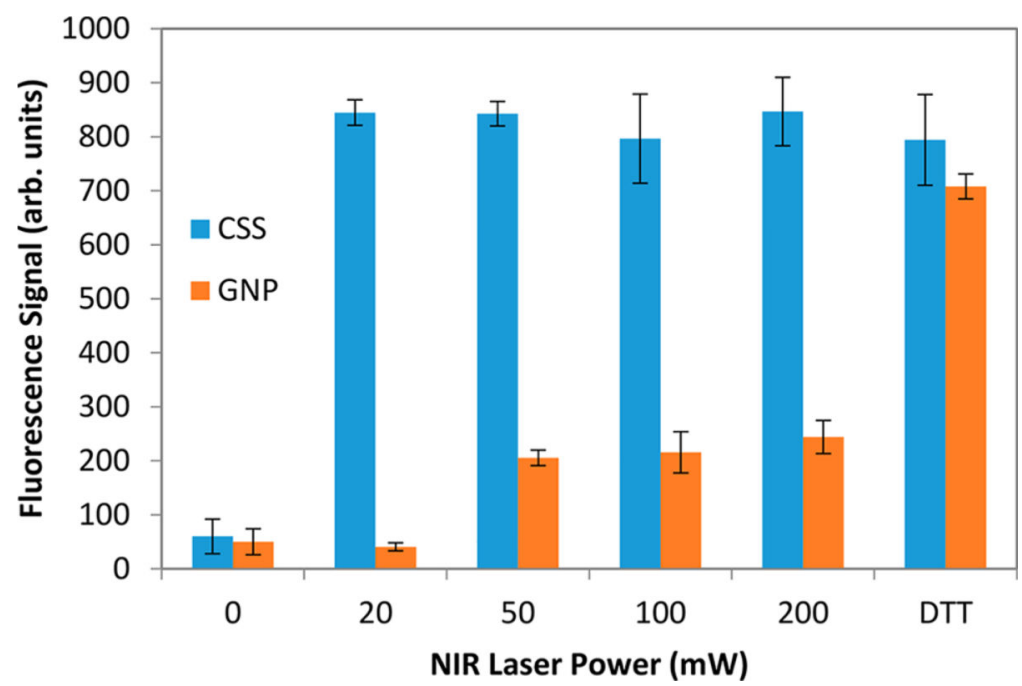

Figure 7.

Fluorescence signals of siRNA-functionalized CSS nanoparticles (blue) and siRNAfunctionalized GNPs after DTT reduction and after NIR laser irradiation at various powers. 


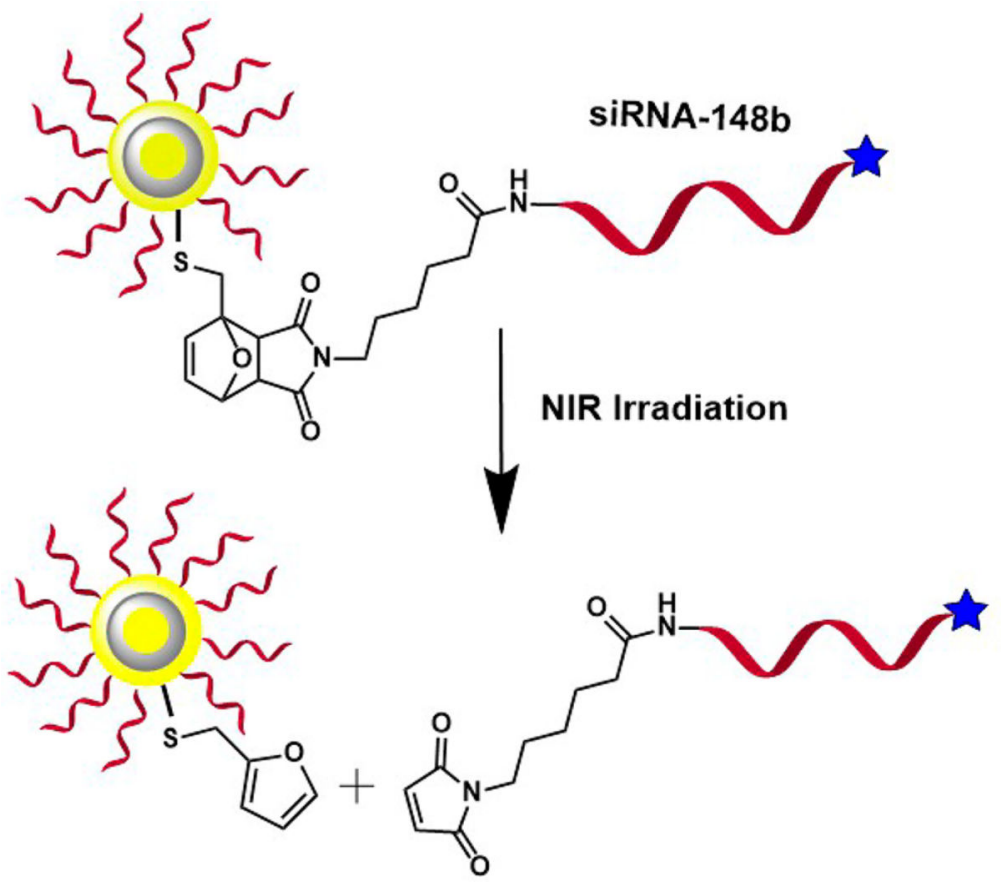

Scheme 1.

SiRNA is Attached to the Surface of Gold-Silver-Gold Core-Shell-Shell Nanoparticles Using Diels-Alder Chemistry ${ }^{\mathrm{a}}$

a NIR irradiation causes photothermal cleaving and siRNA release. 